\title{
Neutrophil Gelatinase-Associated Lipocalin and Retinol-Binding Protein-4 as Biomarkers for Diabetic Kidney Disease
}

\author{
Fatemeh Abbasi $^{a} \quad$ Fatemeh Moosaie $^{a}$ Pegah Khaloo $^{a}$ \\ Fatemeh Dehghani Firouzabadi ${ }^{a}$ Seyede Marzie Fatemi Abharib \\ Bahar Atainia $^{a} \quad$ Maryam Ardeshir $^{a} \quad$ Manouchehr Nakhjavania \\ Alireza Esteghamati ${ }^{a}$ \\ aEndocrinology and Metabolism Research Center, Vali-Asr Hospital, School of Medicine, \\ Tehran University of Medical Sciences, Tehran, Iran; ${ }^{b}$ Department of Pediatrics, Imam Ali \\ Hospital, Alborz University of Medical Sciences, Karaj, Iran
}

\section{Keywords}

Neutrophil gelatinase-associated lipocalin · Retinol-binding protein 4 - Diabetes type 2 .

Albuminuria Chronic kidney disease

\begin{abstract}
Aims: This study was designed to evaluate the conflicting association between 2 tubular protein markers including neutrophil gelatinase-associated lipocalin (NGAL) and retinol-binding protein-4 (RBP-4) with albuminuria and glomerular filtration rate (GFR) and calculate the accuracy of the role of NGAL and RBP-4 in diagnosis of diabetic nephropathy (DN) in patients with type 2 diabetes. Methods: This is a cross-sectional study that included 133 patients with type 2 diabetes. There were 3 diabetic study groups with normoalbuminuria, moderately increased albuminuria, severely increased albuminuria, and non-diabetic control group without any renal disease. We analyzed the difference of urinary NGAL (UNGAL) and RBP-4 between nondiabetics and diabetics, as well as within the diabetic group. We also assessed the association between albuminuria and NGAL and RBP-4. Results: The urinary levels of NGAL and RBP4 were higher in patients with type 2 diabetes compared to nondiabetics as well as in albuminuric diabetics compared to nonalbuminuric patients with diabetes ( $p$ value <0.001). These 2 proteins were higher in patients with severely increased albuminuria compared to patients with moderately increased albuminuria, even after adjustment for other metabolic factors (all $p<$ 0.01). Moreover, areas under the curve of NGAL and RBP-4 for the diagnosis of chronic kidney disease were 80.6 and $74.6 \%$, respectively. Conclusion: UNGAL and RBP-4 are potential markers of tubular damage that may increase before the onset of glomerular markers such as albuminuria and GFR in patients with type 2 diabetes. Therefore, these markers can be used as complementary measurements to albuminuria and GFR in the earlier diagnosis of DN.
\end{abstract}




\section{Kidney \\ Blood Pressure \\ Research}

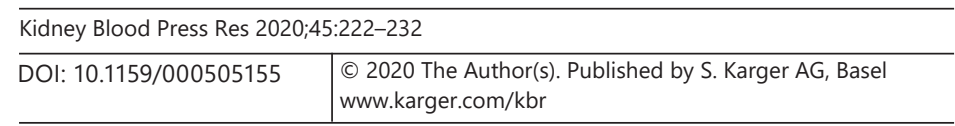

Abbasi et al.: NGAL and RBP-4 as Biomarkers for DKD

\section{Introduction}

Diabetic kidney disease (DKD), which is one of the most common microvascular complications of diabetes, accounts for not only the leading cause of end-stage renal disease but also causes major morbidity and mortality in patients with type 2 diabetes [1-3]. In Iran, the prevalence of nephropathy among the clinically registered adults with diabetes was estimated to be $16.70 \%$ in women and $17.60 \%$ in men [4]. Recent studies have represented that diabetic nephropathy (DN) can begin even in the early stage of diabetes [5]. Although albuminuria and glomerular filtration rate (GFR) are currently accepted standards for diagnosing the onset or progression of DN, some patients with DKD at early stages may show normal urinary albumin level or normal estimated GFR (eGFR) [6]. These conflicting ideas about a diagnostic marker for early detection of renal pathological changes in diabetes have prompted the consideration of the role of other biomarkers. So that, multiple tubular damage markers currently have been detected that could contribute to the early pathogenesis of DKD [2]. Our study was conducted to evaluate 2 of these biomarkers which are increased in response to tubular injury such as neutrophil gelatinase-associated lipocalin (NGAL) and retinol-binding protein 4 (RBP-4).

NGAL is a $25 \mathrm{kDa}$ protein from the lipocalin family. It is produced by neutrophils and injured nephron epithelial cells. NGAL is specifically released into blood and urine as a reaction to nephron injury. Higher urinary levels of NGAL were reported in patients with diabetes in comparison to healthy nondiabetic individuals [6]. Several studies have suggested that NGAL is a specific marker for acute kidney injury as well as early detection of DN [7-9].

RBP-4 is also a $21 \mathrm{kDa}$ protein belonging to the lipocalin superfamily which is almost completely reabsorbed by proximal tubular cells after glomerular filtration. Therefore, urinary RBP-4 has been identified as a very sensitive biomarker for proximal tubular cells dysfunction. Previous studies have shown not only increasing serum and urinary levels of RBP-4 in patients with diabetes but also a determining relationship between urinary RBP-4 and the progression of chronic kidney disease (CKD) in the severely increased albuminuric patients with diabetes. However, the occurrence of normal and moderately increased albuminuria with RBP-4 levels is yet controversial [1].

Most of previous studies have shown a positive correlation between urinary levels of potential tubular injury markers such as NGAL and RBP-4 and glomerular injury markers such as albuminuria [1, 8, 10-15], but there are conflicting reports about the correlation between NGAL and RBP-4 levels with GFR. On the other hand, some studies have shown a significant negative correlation between NGAL and GFR, while others have revealed an independent relationship between these 2 [16-21]. The controversies among the previous studies urged us to measure the levels of urinary NGAL (uNGAL) and RBP-4 in patients with type 2 diabetes who had normal, moderately increased, or severely increased albuminuria. We aimed to explore the relationship between NGAL and RBP-4 as tubular injury markers with GFR and different stages of diabetic albuminuria as well as evaluating the accuracy of these 2 tubular injury markers in diagnosing CKD in patients with type 2 diabetes.

\section{Material and Methods}

\section{Study Population}

This cross-sectional study was conducted in the Diabetes Clinic of Vali-Asr Hospital, a teaching hospital affiliated with Tehran University of Medical Sciences (Tehran, Iran) from 2018 to 2019. One hundred and thirty-three patients with diabetes were recruited 


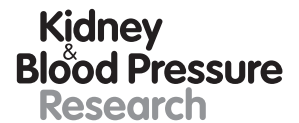

Kidney
Blood Pressure
Research

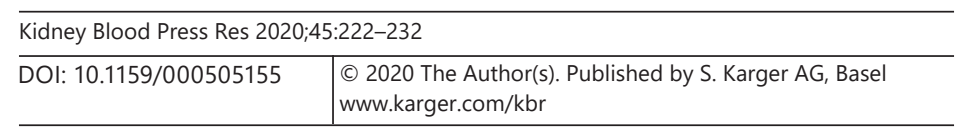

Abbasi et al.: NGAL and RBP-4 as Biomarkers for DKD

and categorized into 3 groups: 44 patients with normal albumin excretion (albumin/ creatinine ratio $[\mathrm{ACR}]<30 \mathrm{mg} / \mathrm{g}$ creatinine [Cr]), 45 patients with moderately increased albuminuria (ACR 30-300 mg/g Cr), and 44 patients with severely increased albuminuria (ACR $>300 \mathrm{mg} / \mathrm{g} \mathrm{Cr}$ ). Moreover, we recruited 39 healthy participants as the control group. The diagnosis of diabetes was made based on the American Diabetes Association diagnostic criteria (2014). We included patients with type 2 diabetes with no other cause for nephropathy other than diabetes, no inflammatory, rheumatologic or neoplastic illness, no use of glucocorticoids, without symptomatic heart failure, with normal liver function and with $\mathrm{Cr}>2$. Exclusion criteria were smoking, pregnancy, congestive heart failure, hypertension or use of anti-hypertension medications, alternation in leukocyte count, renal transplant, severe liver dysfunction, lupus nephritis, nephrotoxic drugs, insulin therapy, malignancies, infectious diseases, and hospital admission in the recent months. The study protocol was approved by the local Ethics Committee of the Tehran University of Medical Sciences. Written informed consent was obtained from all participants prior to the study.

\section{Clinical and Laboratory Measurements}

All participants' weight and height were measured, and their body mass index (BMI) was determined $\left(\mathrm{kg} / \mathrm{m}^{2}\right)$. Waist circumference (WC) was also measured (in centimeters). Venous blood samples were collected from each participant after a 12-h overnight fasting for biochemical analysis. Serum fasting blood sugar (FBS) and 2-h post-prandial (2HPP) glucose were determined by the glucose oxidase method based on Trinder [22]. For the assessment of glycated hemoglobin A1c (HbA1c), high-performance liquid chromatography was performed according to the method of Lahousen et al. [23]. Plasma lipid profile including serum total cholesterol [24], triglyceride, high-density lipoprotein cholesterol (HDL-C) [25], and low-density lipoprotein cholesterol (LDL-C) [26] were determined by using enzymatic method with commercially available kits (Pars Azmoon, Karaj, Iran). Measurement of serum $\mathrm{Cr}$ was performed by enzymatic method. Urinary albumin excretion was measured by calorimetric methods using commercial kits (ZiestChem Diagnostics, Tehran, Iran). NGAL and RBP-4 were both measured using Enzyme-Linked Immunosorbent Assay kit (Bioassay Technology, intra-assay CV $<8 \%$, inter-assay CV $<$ $10 \%)$. GFR was calculated using the CKD-EPI method [27]. Based on the KDIGO, CKD was defined as kidney damage or GFR $<60 \mathrm{~mL} / \mathrm{min} / 1.73 \mathrm{~m}^{2}$ for 3 months or more, irrespective of cause.

\section{Statistical Analysis}

STATA software version 12 for Windows (Stata Corp., College Station, TX, USA) was used for statistical analysis. Continuous variables were demonstrated as mean \pm SD. Categorical outcomes are introduced as proportions. Design-based parametric independent $t$ test or nonparametric Mann-Whitney U Test was used to compare continuous data between the binary variables. Multiple Logistic regression models were used to examine the association of albuminuria with NGAL and RBP-4. This association was adjusted for age and sex in model 1 and for age, sex, WC, FBS, 2HPP, HbA1c, cholesterol, HDL, LDL, TG, and BMI in model 2. $p$ value $<0.05$ was considered statistically significant. We used model 2 to assess the association between GFR, NGAL, and RBP-4. Multiple linear regression models adjusted for duration of diabetes, FBS, HbA1c, HDL, LDL, total cholesterol, and BMI were used to examine the association of GFR with NGAL and RBP. The C-statistics was used to assess the discriminatory power of NGAL and RBP-4 for prediction of CKD in patients with type 2 diabetes. 


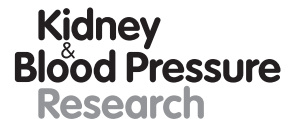

Abbasi et al.: NGAL and RBP-4 as Biomarkers for DKD

Table 1. Clinical and biochemical characteristics of patients; significant differences have been reported according to 3 references (control, normal albuminuria, and moderately increased albuminuria)

\begin{tabular}{lcccc}
\hline & $\begin{array}{l}\text { Control } \\
(n=39)\end{array}$ & $\begin{array}{l}\text { Normal } \\
\text { albuminuria } \\
(n=44)\end{array}$ & $\begin{array}{l}\text { Moderately increased } \\
\text { albuminuria } \\
\text { (micro albuminuria) } \\
(n=45)\end{array}$ & $\begin{array}{l}\text { Severely increased } \\
\text { albuminuria } \\
\text { (macro albuminuria) } \\
(n=44)\end{array}$ \\
\hline Gender, female, $n$ (\%) & & & $17(37.77)$ & $20(45.45)$ \\
Age, years, mean \pm SD & $9(23.07)$ & $26(59.09)$ & $53.71 \pm 9.40^{*}$ & $58.16 \pm 6.88^{* \$ \#}$ \\
BMI, kg/m ${ }^{2}$, mean \pm SD & $48.13 \pm 5.3$ & $51.81 \pm 7.04$ & $29.23 \pm 3.74^{*}$ & $28.16 \pm 3.66$ \\
WC, cm, mean \pm SD & $27.21 \pm 3.21$ & $28.15 \pm 3.44$ & $100.04 \pm 9.6$ & $98.48 \pm 7.30$ \\
Duration of diabetes, years, mean \pm SD & $97.77 \pm 7.74$ & $97.68 \pm 7.8$ & $15.05 \pm 6.27$ & $16.48 \pm 5.41$ \\
FBS, mg/dL, mean \pm SD & $92.77 \pm 7.95$ & $144.48 \pm 30.9^{*}$ & $167.68 \pm 44.48^{* \#}$ & $168.61 \pm 54.15^{* \#}$ \\
HbA1c, $\%$, mean \pm SD & $4.68 \pm 0.57$ & $7.23 \pm 1.08$ & $8.09 \pm 1.02$ & $10.0 \pm 1.27^{*}$ \\
2HPP, mg/dL, mean \pm SD & $100.92 \pm 4.5$ & $203.52 \pm 54.9^{*}$ & $239.24 \pm 74.97^{*}$ & $235.02 \pm 88.7^{*}$ \\
TG, mg/dL, mean \pm SD & $157.54 \pm 73.4$ & $158.64 \pm 81.1$ & $196.87 \pm 130.9$ & $158.8 \pm 65.7$ \\
Cholesterol, mg/dL, mean \pm SD & $181 \pm 44.7$ & $175.57 \pm 38.4$ & $183.82 \pm 39.44$ & $173.54 \pm 35.9$ \\
LDL-C, mg/dL, mean \pm SD & $116.5 \pm 33.4$ & $101.57 \pm 29.6$ & $105.91 \pm 32.8$ & $97.89 \pm 30.06^{*}$ \\
HDL-C, mg/dL, mean \pm SD & $45.4 \pm 11.3$ & $44.30 \pm 8.83$ & $46.20 \pm 20.8$ & $45.27 \pm 10.36$ \\
GFR, mean \pm SD & $99.5 \pm 11.02$ & $86.19 \pm 12.93^{*}$ & $72.06 \pm 11.69^{* \#}$ & $40.41 \pm 6.03^{* \# \$}$ \\
Urine albumin, mean \pm SD & $4.15 \pm 1.87$ & $10.24 \pm 4.5^{*}$ & $79.86 \pm 35.7^{* \#}$ & $415.75 \pm 102.9^{* \# \$}$ \\
NGAL, mean \pm SD & $77.02 \pm 8.9$ & $114.08 \pm 16.38^{*}$ & $122.17 \pm 13.25^{* \#}$ & $129.13 \pm 13.6^{* *}$ \\
RBP-4, mean \pm SD & $34.17 \pm 7.8$ & $43.64 \pm 10.8^{*}$ & $49.58 \pm 10.43^{* \#}$ & $52.47 \pm 8.00^{* \#}$ \\
\hline
\end{tabular}

${ }^{*} p<0.05$ versus control, ${ }^{\#} p<0.05$ versus normal albuminuria, ${ }^{\$} p<0.05$ versus moderately increased albuminuria.

BMI, body mass index; WC, waist circumference; FBS, fasting blood sugar; HbA1c, hemoglobin; 2HPP, 2-h post-prandial; LDL-C, low-density lipoprotein cholesterol; HDL-C, glycated high-density lipoprotein cholesterol; GFR, glomerular filtration rate; NGAL, neutrophil gelatinase-associated lipocalin; RBP-4, retinol-binding protein-4; TG, triglyceride.

\section{Results}

Basic Characteristics of Diabetic Patients and Control Group

One hundred and thirty-three patients including 72 females $(40.7 \%)$ were enrolled in this study. Patients with diabetes were classified into 3 groups based on their ACR. Forty-four patients were counted for normal albuminuria group (26 females; mean age $51.81 \pm 7.04$ ), moderately increased albuminuria (microalbuminuria) group consisted of 45 patients (17 females; mean age $53.71 \pm 9.40$ ), and 44 patients were accounted for severely increased albuminuria (macroalbuminuria) group ( 20 females; mean age $58.16 \pm 6.88$ ). Other baseline characteristics of the patients enrolled in this study are presented in Table 1. Lipid profile showed no overall significant association with albuminuria, except for LDL-C, which was significantly different in patients with severely increased albuminuria as compared to the control group $(97.89 \pm 30.06,116.5 \pm 33.4$, respectively, $p$ value $=0.049)$. GFR was significantly lower among patients with severely increased albuminuria compared to the control group and those with normal and moderately increased albuminuria (40.41 \pm 6.03 vs. $99.5 \pm 11.02,86.19 \pm 12.93$, $72.06 \pm 11.69$, respectively).

\section{Univariate Correlation for NGAL and RBP-4 and Multiple Regression Models}

Urine levels of NGAL and RBP-4 were significantly higher in all 3 groups of patients with diabetes compared to the control group (Table 1). In addition, patients with severely increased albuminuria had higher levels of NGAL and RBP-4 compared to the patients with normal albuminuria ( $129.13 \pm 13.6$ vs. $114.08 \pm 16.38,52.47 \pm 8.00$ vs. $43.64 \pm 10.8$, respectively), but there was no statistically significant difference between the severely increased albuminuria 
Table 2. Relation between urinary NGAL and RBP-4 and albuminuria

\begin{tabular}{llllll}
\hline & NGAL & & & RBP-4 \\
\cline { 2 - 3 } \cline { 5 - 6 } & model 1 & model 2 & model 1 & model 2 \\
\hline Control & Reference & Reference & & Reference & Reference \\
Normal albuminuria & $35.3(<0.001)$ & $34.26(<0.001)$ & & $10.50(<0.001)$ & $11.23(<0.001)$ \\
Moderately increased albuminuria & $43.99(<0.001)$ & $43.42(<0.001)$ & & $16.52(<0.001)$ & $17.61(<0.001)$ \\
Severely increased albuminuria & $50.16(<0.001)$ & $48.38(<0.001)$ & & $20.26(<0.001)$ & $21.99(<0.001)$ \\
\hline Normal albuminuria & Reference & Reference & & Reference & Reference \\
Moderately increased albuminuria & $8.59(0.003)$ & $9.17(0.003)$ & & $6.02(0.003)$ & $6.38(0.003)$ \\
Severely increased albuminuria & $14.76(<0.001)$ & $14.12(<0.001)$ & & $9.75(<0.001)$ & $10.77(<0.001)$ \\
\hline Moderately increased albuminuria & Reference & Reference & & Reference & Reference \\
Severely increased albuminuria & $6.17(0.035)$ & $4.95(0.097)$ & & $3.73(0.068)$ & $4.38(0.037)$ \\
\hline
\end{tabular}

Data are presented as $\beta$ coefficient ( $p$ value).

Model 1: adjusted for age, sex; Model 2: adjusted for age, sex, WC, FBS, 2HPP, hbA1C, cholesterol, HDL, LDL, TG, BMI.

WC, waist circumference; FBS, fasting blood sugar; 2HPP, 2-h postprandial; HbA1c, hemoglobin A1c; HDL, high-density lipoprotein; LDL, low-density lipoprotein; TG, triglyceride; BMI, body mass index; NGAL, neutrophil gelatinase-associated lipocalin; RBP-4, retinol-binding protein-4.

Table 3. Multiple logistic regression analysis determining the association between eGFR, DKD, and NGAL and RBP-4 in patients with type 2 diabetes

\begin{tabular}{lll}
\hline Biomarkers & eGFR & DKD \\
\hline NGAL & $-0.2(-0.375$ to -0.25$), 0.02$ & $0.05(0.01$ to 0.09$), 0.012$ \\
RBP-4 & $-1.06(-0.213$ to -0.001$), 0.05$ & $0.08(0.03$ to 0.13$), 0.002$ \\
\hline
\end{tabular}

Data are presented as coefficient $(95 \% \mathrm{CI}), p$ value.

NGAL, neutrophil gelatinase-associated lipocalin; RBP-4, retinol-binding protein-4; eGFR, estimated glomerular filtration rate; DKD, diabetic kidney disease.

and moderately increased albuminuria groups $(129.13 \pm 13.6$ vs. $122.17 \pm 13.25,52.47 \pm 8.00$ vs. $49.58 \pm 10.43$, respectively; Table 1 ).

Two models were considered; model 1 was adjusted for age and sex and model 2 was adjusted for all confounding variables such as age, sex, WC, FBS, 2HPP, HbA1c, cholesterol, HDL, LDL, TG, and BMI as listed in Table 1. Both models showed a statistically significant difference of NGAL and RPB levels among the patients with diabetes compared to the control group and also among diabetics with moderately and severely increased albuminuria compared to diabetics with normal levels of urinary albumin (Table 2). However, when analyzing the difference of these 2 biomarkers between patients with moderately and severely increased albuminuria, the 2 models revealed different results. UNGAL levels were significantly higher among diabetic patients with severely increased albuminuria compared to diabetic patients with moderately increased albuminuria in the first model (50.16 vs. 43.99), while RBP-4 revealed the same significant trend in the second model (21.99 vs. 17.61; Table 2 ). Also, the result of logistic regression model, for CKD is provided in Table 3. Results from this study showed a positive correlation between NGAL, RBP-4, and CKD with regression coefficients of $0.50(p$ value $=0.012)$ and $0.08(p$ value $=0.002)$, respectively. As well as negative correlation among NGAL, RBP-4 and eGFR with regression coefficient of 0.2 ( $p$ value $=0.02)$ and $1.06(p$ value $=0.05)$, respectively $($ Table 3$)$. 


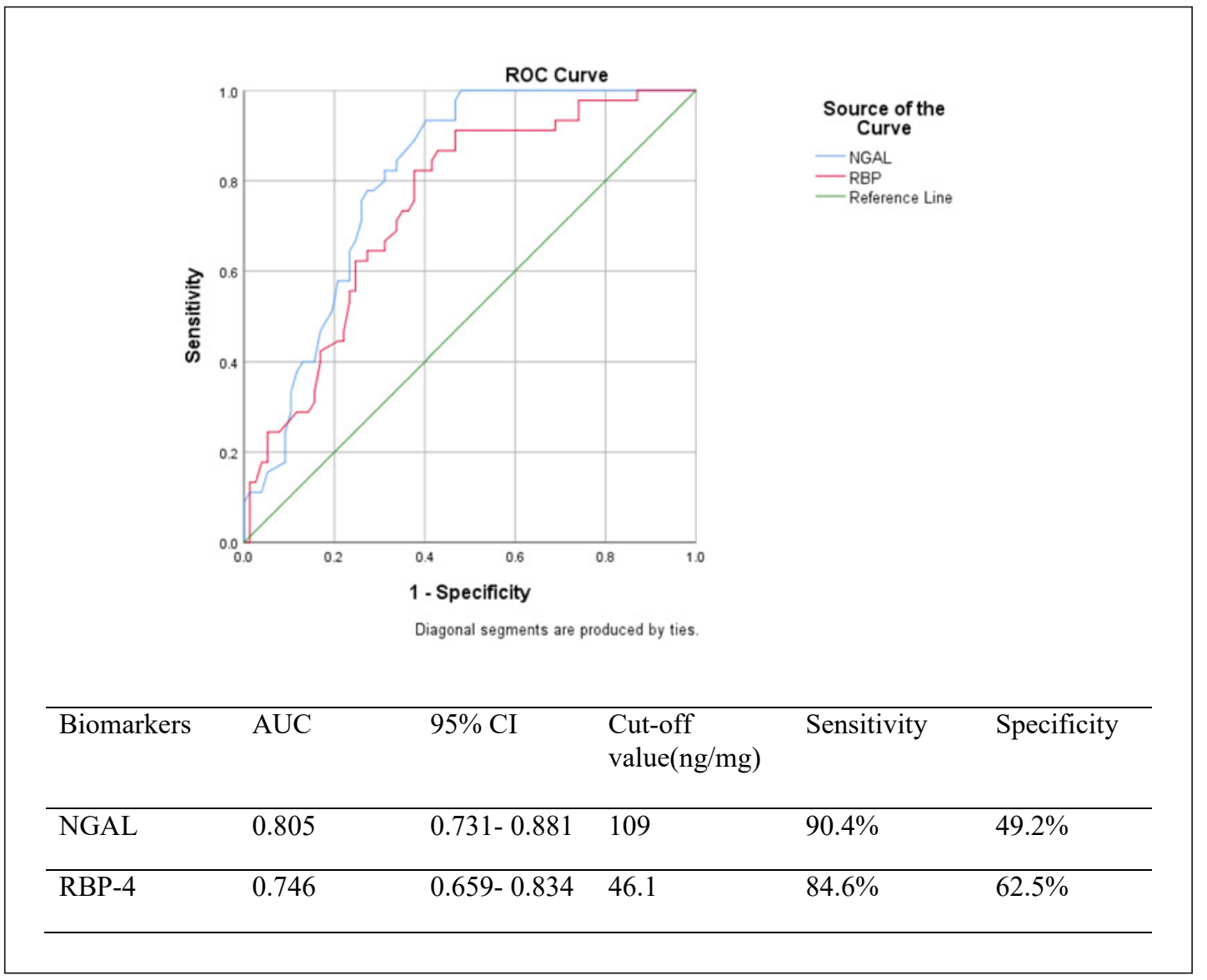

Fig. 1. ROC curve of NGAL and RBP-4 for CKD diagnosis; the AUC and the cutoff value of each urinary biomarker are presented in the separate table under the figure. AUC, area under the ROC curve; NGAL, neutrophil gelatinase-associated lipocalin; RBP-4, retinol-binding protein 4; ROC, receiver operating characteristic.

Receiver Operating Characteristic Curve Analysis of NGAL and RBP-4

For determining the diagnostic index of NGAL and RBP-4 in early detection of DN in patients with normal urinary albumin level and eGFR, receiver operating characteristic curve analyses were performed. The sensitivity and specificity for NGAL was calculated as 90.4 and $49.2 \%$ respectively. The sensitivity and specificity for RBP-4 were calculated as 84.6 and $62.5 \%$, respectively. The analysis showed that these 2 proteins are rather sensitive than specific. Area under the curve (AUC) for NGAL was calculated as $80.5 \%$ with a cutoff value of 109 . AUC for RBP-4 was calculated as $74.6 \%$ with cutoff value of 46.1 . According to our analysis, NGAL is good, and RBP-4 is a fair complementary diagnostic tool for early detection of DKD (Fig. 1).

\section{Discussion}

Early diagnosis and prevention of DN which is the leading cause of morbidity and mortality among patients with diabetes and accounts for enormous medical expenses worldwide is of outmost importance. Our study has determined the diagnostic profile of the urine level of NGAL and RBP-4 in early detection of advanced DKD even before changing glomerular markers such as increasing albuminuria and decreasing GFR levels. Although albuminuria and low GFR have 


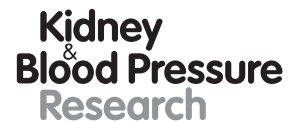

Kidney
Blood Pressure

Research \begin{tabular}{l|l}
\hline Kidney Blood Press Res 2020;45:222-232 \\
\hline DOI: 10.1159/000505155 & $\begin{array}{l}\text { @ 2020 The Author(s). Published by S. Karger AG, Basel } \\
\text { www.karger.com/kbr }\end{array}$ \\
\hline
\end{tabular}

Abbasi et al.: NGAL and RBP-4 as Biomarkers for DKD

been the 2 most common markers for diagnosis of DN so far, there are several limitations to the measurement of albuminuria as a biomarker of DKD. First of all, albuminuria can be increased as a result of comorbidities of diabetes (e.g., obesity and hypertension), making it a less specific marker for diagnosis of DKD [28]. Second, albuminuria is not observed in 30-40\% of patients with type 2 diabetes and kidney disease [29]. Third, hypertension is often treated with reninangiotensin-aldosterone inhibitors which reduce the GFR, and thus albuminuria is normalized [30]. Tubular damage in $30 \%$ of type 2 diabetics with DN occurs in advance to glomerular damage [31]. This has prompted many researchers to look for and assess the diagnostic accuracy of surrogate markers for DKD. uNGAL and RBP- 4 are 2 proteins that can be suggestive of tubular damage [32]. NGAL binds with iron forming NGAL:siderophore:Fe3b complex which is essential for nephron formation [33]. In addition, NGAL has significant kidney protective activities through increasing proliferative state and reducing apoptosis [34]. Synthesis of NGAL is upregulated in the distal and proximal tubule cells as a result of ischemia in response to kidney injury $[35,36]$. This is the reason why NGAL is quickly increased in the urine in response to tubular injury. Also, RBP-4 is a specific carrier for retinol (vitamin A alcohol) in the blood that is produced by liver and fat cells [37]. Elevated urine level of this marker that is completely reabsorbed in the proximal tubule accounts for proximal tubular cells damage in patients with DKD [16]. There are several possible explanations for this increase. One of them could be that urinary RBP might reflect tubule-interstitial fibrosis since its excretion is directly related to the proximal tubular cells function. Another possible explanation is that elevated urinary RBP could be due to the impairment in one of the mechanisms that indirectly affects proximal tubular cells function (i.e., impairment in reabsorption machinery, glomerular filtration barrier, massive production of low molecular weight protein) [17]. Also, associations have been found between RBP and insulin resistance, which may be due to an impaired insulin signaling pathways in skeletal muscle [38].

Various studies have investigated the association between albuminuria and the level of NGAL and RBP-4 in the urine of patients with and without type 2 diabetes. Many previous studies indicate that urine NGAL and RBP-4 levels are higher in patients with diabetes once compared to control groups as well as they are positively correlated with albuminuria in diabetic patients $[1,8,10,12-16,39,40]$. Moreover, urine NGAL and RBP-4 are significantly greater in diabetic patients with moderately increased albuminuria in comparison to patients with normal albuminuria and control groups and are positively correlated with urine ACR [1, $19,20,32,41$ ]. Also, Wu et al. [1] realized that both NGAL and RBP-4 were independently associated with albuminuria. Of notable mention, some studies found out that uNGAL and RBP-4 were higher in normoalbuminuric diabetics compared to control groups. This implies the possible role of urinary level of NGAL and RBP-4 as predictors for early detection of DN as it warns tubular damage which precedes moderately increased albuminuria via glomerular damage $[13,16,32,42]$. Some studies defined that NGAL could be an indicative of not only the onset of DN but also the rate of DN progression [6,43]. Also, RBP-4 could be used for follow-up of patients with DN to monitor the progression of their disease [16, 44].

Findings from this study showed statistically significant higher UNGAL and RBP-4 levels in patients with type 2 diabetes in comparison to the healthy control group. These 2 proteins were higher not only in albuminuric diabetic patients compared to nonalbuminuric diabetic patients but also in diabetic patients with severely increased albuminuria once compared to patients with moderately increased albuminuria.

Various studies have evaluated the association between UNGAL and GFR in patients with type 2 diabetes. The results from the studies on this issue are conflicting as Satirapoj [43] in a recent review article suggested that an increase in urinary level of NGAL is independent of a decrease in GFR. Also, Rotbain Curovic et al. [9] have recently shown that NGAL is not a predictor of GFR. Moreover, a few studies have attempted to assess the association between urinary RBP-4 levels and GFR that have determined the negative correlation between them 


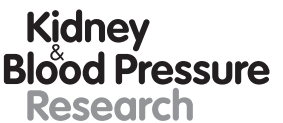

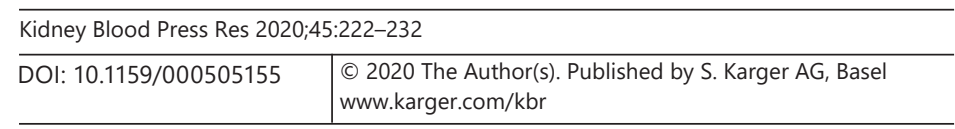

Abbasi et al.: NGAL and RBP-4 as Biomarkers for DKD

in non-diabetic patients with CKD [17]. In addition, some other studies had similar results to ours, indicating a statistically significant negative correlation between both NGAL and RBP-4 levels with GFR [16, 18-21] (Table 3).

Previous studies similar to ours assessed diagnostic accuracy of the urine level of NGAL and RBP-4 as biomarkers of DKD in patients with type 2 diabetes. Most of them have presented AUC of $80 \%$ or above for both factors which was significant [11,16, 19, 45]. Furthermore, our result showed that AUCs of NGAL and RBP-4 for the diagnosis of CKD in type 2 diabetic patients with DN were 87.5 and $88.7 \%$, respectively. The AUC could determine valuable statistical comparison of diagnostic tests [46].

According to the positive correlation of urine levels of NGAL and RBP-4 with albuminuria and the high diagnostic accuracy of these 2 biomarkers for early detecting of CKD in type 2 diabetic patients, our findings imply that NGAL and RBP-4 could be used as complementary measurements to albuminuria in early diagnosis of CKD in patients with type 2 diabetes.

One of the strengths of this study that helped boost the discriminative strength of the markers and generalization of the findings is the inclusion of both diabetic and nondiabetic patients as well as classification of diabetic patients into 3 groups with normal albuminuria, moderately increased albuminuria, and severely increased albuminuria. We were able to analyze the difference of NGAL and RBP-4 between nondiabetics and diabetics as well as within the diabetic group. Moreover, this is one of the very few studies measuring both NGAL and RBP-4 simultaneously.

Cross-sectional nature of the study and relatively low sample size were limitations of this study. This study has a cross-sectional design. Whether the damage markers, as assessed in our study, predict the progression of DN has to be investigated in large, long-term, prospective, observational studies. Moreover, we have only measured the levels of NGAL and RBP-4 in the 24-h urine sample. Measurement of serum levels of these 2 proteins is suggested in the future studies to further explore the role of NGAL and RBP-4 in the diagnosis and prognosis of DN. Lastly, although potential confounders have been adjusted, residual confounding may exist due to measurement errors or unmeasured factors.

\section{Conclusion}

UNGAL and RBP-4 may serve as efficient biomarkers of tubular damage, and thus could potentially be used as complementary measurements to the conventional approaches for diagnosis of DN in patients with type 2 diabetes. Further long-term, prospective, observational studies should be deigned to determine the association between these 2 markers and the progression of DN.

\section{Acknowledgments}

Authors wish to thank patients for their participation and kind cooperation.

\section{Statement of Ethics}

All procedures performed in studies involving human participants were in accordance with the ethical standards of the institutional and/or national research committee and with the 1964 Helsinki Declaration and its later amendments or comparable ethical standards. Informed consent was obtained from all individual participants included in the study. 


\section{Kidney \\ Blood Pressure Research}

\begin{tabular}{l|l}
\hline Kidney Blood Press Res 2020:45:222-232 \\
\hline DOI: 10.1159/000505155 & $\begin{array}{l}\text { @ 2020 The Author(s). Published by S. Karger AG, Basel } \\
\text { www.karger.com/kbr }\end{array}$ \\
\hline
\end{tabular}

Abbasi et al.: NGAL and RBP-4 as Biomarkers for DKD

\section{Disclosure Statement}

The authors declare that they have no competing interest.

\section{Funding Sources}

This research did not receive any specific grant from funding agencies in the public, commercial, or not-for-profit sectors.

\section{Author Contributions}

A.E., M.N., and F.A.: conception or design of the work. F.M., S.M.F.A., and F.D.F: drafting the article. P.K., F.M., B.A., and M.A.: data analysis and interpretation. A.E.: critical revision of the article. All the authors approved the final version and have the agreement to be accountable for all aspects of the work in ensuring that questions related to the accuracy or integrity of any part of the work are appropriately investigated and resolved.

\section{Data Availability}

All data generated or analyzed during this study will be made available on request.

\section{References}

1 Wu J, Shao X, Lu K, Zhou J, Ren M, Xie X, et al. Urinary RBP and NGAL Levels are Associated with Nephropathy in Patients with Type 2 Diabetes. Cell Physiol Biochem. 2017;42(2):594-602.

2 Tang XY, Zhou JB, Luo FQ, Han YP, Zhao W, Diao ZL, et al. Urine NGAL as an early biomarker for diabetic kidney disease: accumulated evidence from observational studies. Ren Fail. 2019 Nov; 41(1):446-54.

3 Noshad S, Afarideh M, Heidari B, Mechanick JI, Esteghamati A. Diabetes Care in Iran: Where We Stand and Where We Are Headed. Ann Glob Health. 2015 Nov-Dec;81(6):839-50.

4 Esteghamati A, Larijani B, Aghajani MH, Ghaemi F, Kermanchi J, Shahrami A, et al. Diabetes in Iran: Prospective Analysis from First Nationwide Diabetes Report of National Program for Prevention and Control of Diabetes (NPPCD-2016). Sci Rep. 2017 Oct;7(1):13461.

5 Garg V, Kumar M, Mahapatra HS, Chitkara A, Gadpayle AK, Sekhar V. Novel urinary biomarkers in pre-diabetic nephropathy. Clin Exp Nephrol. 2015 Oct;19(5):895-900.

6 Nauta FL, Boertien WE, Bakker SJ, van Goor H, van Oeveren W, de Jong PE, et al. Glomerular and tubular damage markers are elevated in patients with diabetes. Diabetes Care. 2011 Apr;34(4):975-81.

7 Singer E, Markó L, Paragas N, Barasch J, Dragun D, Müller DN, et al. Neutrophil gelatinase-associated lipocalin: pathophysiology and clinical applications. Acta Physiol (Oxf). 2013 Apr;207(4):663-72.

8 Kaul A, Behera MR, Rai MK, Mishra P, Bhaduaria DS, Yadav S, et al. Neutrophil Gelatinase-associated Lipocalin: As a Predictor of Early Diabetic Nephropathy in Type 2 Diabetes Mellitus. Indian J Nephrol. 2018 Jan-Feb; 28(1):53-60.

9 Rotbain Curovic V, Hansen TW, Eickhoff MK, von Scholten BJ, Reinhard H, Jacobsen PK, et al. Urinary tubular biomarkers as predictors of kidney function decline, cardiovascular events and mortality in microalbuminuric type 2 diabetic patients. Acta Diabetol. 2018 Nov;55(11):1143-50.

10 De Muro P, Lepedda AJ, Nieddu G, Idini M, Tram Nguyen HQ, Lobina O, et al. Evaluation of Early Markers of Nephropathy in Patients with Type 2 Diabetes Mellitus. Biochem Res Int. 2016;2016:7497614.

11 Zeng XF, Lu DX, Li JM, Tan Y, Li Z, Zhou L, et al. Performance of urinary neutrophil gelatinase-associated lipocalin, clusterin, and cystatin $\mathrm{C}$ in predicting diabetic kidney disease and diabetic microalbuminuria: a consecutive cohort study. BMC Nephrol. 2017 Jul;18(1):233.

12 Bolignano D, Lacquaniti A, Coppolino G, Donato V, Fazio MR, Nicocia G, et al. Neutrophil gelatinase-associated lipocalin as an early biomarker of nephropathy in diabetic patients. Kidney Blood Press Res. 2009;32(2):91-8.

13 Papadopoulou-Marketou N, Margeli A, Papassotiriou I, Chrousos GP, Kanaka-Gantenbein C, Wahlberg J. NGAL as an Early Predictive Marker of Diabetic Nephropathy in Children and Young Adults with Type 1 Diabetes Mellitus. J Diabetes Res. 2017;2017:7526919. 
14 Hong CY, Chia KS, Ling SL. Urinary protein excretion in Type 2 diabetes with complications. J Diabetes Complications. 2000 Sep-Oct;14(5):259-65.

15 Hong CY, Chia KS, Ling SL. Urine protein excretion among Chinese patients with type 2 diabetes mellitus. Med J Malaysia. 2000 Jun;55(2):220-9.

16 Fiseha T, Tamir Z. Urinary Markers of Tubular Injury in Early Diabetic Nephropathy. Int J Nephrol. 2016;2016: 4647685.

17 Domingos MA, Moreira SR, Gomez L, Goulart A, Lotufo PA, Benseñor I, et al. Urinary Retinol-Binding Protein: Relationship to Renal Function and Cardiovascular Risk Factors in Chronic Kidney Disease. PLoS One. 2016 Sep;11(9):e0162782.

18 Wu J, Ding Y, Zhu C, Shao X, Xie X, Lu K, et al. Urinary TNF- $\alpha$ and NGAL are correlated with the progression of nephropathy in patients with type 2 diabetes. Exp Ther Med. 2013 Dec;6(6):1482-8.

19 Mahfouz MH, Assiri AM, Mukhtar MH. Assessment of Neutrophil Gelatinase-Associated Lipocalin (NGAL) and Retinol-Binding Protein 4 (RBP4) in Type 2 Diabetic Patients with Nephropathy. Biomark Insights. 2016 Feb; 11:31-40.

20 Park SE, Lee NS, Park JW, Rhee EJ, Lee WY, Oh KW, et al. Association of urinary RBP4 with insulin resistance, inflammation, and microalbuminuria. Eur J Endocrinol. 2014 Oct;171(4):443-9.

21 Nielsen SE, Reinhard H, Zdunek D, Hess G, Gutiérrez OM, Wolf M, et al. Tubular markers are associated with decline in kidney function in proteinuric type 2 diabetic patients. Diabetes Res Clin Pract. 2012 Jul;97(1): 71-6.

22 Trinder P. Determination of blood glucose using an oxidase-peroxidase system with a non-carcinogenic chromogen. J Clin Pathol. 1969 Mar;22(2):158-61.

23 Lahousen T, Roller RE, Lipp RW, Schnedl WJ. Silent haemoglobin variants and determination of HbA(1c) with the HPLC Bio-Rad Variant II. J Clin Pathol. 2002 Sep;55(9):699-703.

24 Allain CC, Poon LS, Chan CS, Richmond W, Fu PC. Enzymatic determination of total serum cholesterol. Clin Chem. 1974 Apr;20(4):470-5.

25 Lopes-Virella MF, Stone P, Ellis S, Colwell JA. Cholesterol determination in high-density lipoproteins separated by three different methods. Clin Chem. 1977 May;23(5):882-4.

26 Friedewald WT, Levy RI, Fredrickson DS. Estimation of the concentration of low-density lipoprotein cholesterol in plasma, without use of the preparative ultracentrifuge. Clin Chem. 1972 Jun;18(6):499-502.

27 Levey AS, Coresh J, Greene T, Stevens LA, Zhang YL, Hendriksen S, et al.; Chronic Kidney Disease Epidemiology Collaboration. Using standardized serum creatinine values in the modification of diet in renal disease study equation for estimating glomerular filtration rate. Ann Intern Med. 2006 Aug;145(4):247-54.

28 Duran-Perez EG, Almeda-Valdes P, Cuevas-Ramos D, Campos-Barrera E, Muñoz-Hernandez L, Gomez-Perez FJ. Treatment of metabolic syndrome slows progression of diabetic nephropathy. Metab Syndr Relat Disord. 2011 Dec;9(6):483-9.

29 Kim SS, Song SH, Kim IJ, Kim WJ, Jeon YK, Kim BH, et al. Nonalbuminuric proteinuria as a biomarker for tubular damage in early development of nephropathy with type 2 diabetic patients. Diabetes Metab Res Rev. 2014 Nov;30(8):736-41.

30 Foundation NK; National Kidney Foundation. KDOQI clinical practice guideline for diabetes and CKD: 2012 update. Am J Kidney Dis. 2012 Nov;60(5):850-86.

31 Retnakaran R, Cull CA, Thorne KI, Adler AI, Holman RR; UKPDS Study Group. Risk factors for renal dysfunction in type 2 diabetes: U.K. Prospective Diabetes Study 74. Diabetes. 2006 Jun;55(6):1832-9.

32 Zylka A, Dumnicka P, Kusnierz-Cabala B, Gala-Bladzinska A, Ceranowicz P, Kucharz J, et al. Markers of Glomerular and Tubular Damage in the Early Stage of Kidney Disease in Type 2 Diabetic Patients. Mediators Inflamm. 2018 Aug;2018:7659243.

33 Yang J, Goetz D, Li JY, Wang W, Mori K, Setlik D, et al. An iron delivery pathway mediated by a lipocalin. Mol Cell. 2002 Nov;10(5):1045-56.

34 Mishra J, Mori K, Ma Q, Kelly C, Yang J, Mitsnefes M, et al. Amelioration of ischemic acute renal injury by neutrophil gelatinase-associated lipocalin. J Am Soc Nephrol. 2004 Dec;15(12):3073-82.

35 Papadopoulou-Marketou N, Skevaki C, Kosteria I, Peppa M, Chrousos GP, Papassotiriou I, et al. NGAL and cystatin C: two possible early markers of diabetic nephropathy in young patients with type 1 diabetes mellitus: one year follow up. Hormones (Athens). 2015 Apr-Jun;14(2):232-40.

36 Mishra J, Ma Q, Prada A, Mitsnefes M, Zahedi K, Yang J, et al. Identification of neutrophil gelatinase-associated lipocalin as a novel early urinary biomarker for ischemic renal injury. J Am Soc Nephrol. 2003 Oct;14(10): 2534-43.

37 RBP4 retinol binding protein 4 [Homo sapiens (human)] Gene ID: 5950. 2-Jul-2019.

38 Takebayashi K, Suetsugu M, Wakabayashi S, Aso Y, Inukai T. Retinol binding protein-4 levels and clinical features of type 2 diabetes patients. J Clin Endocrinol Metab. 2007 Jul;92(7):2712-9.

39 Hafez MH, El-Mougy FA, Makar SH, Abd El Shaheed S. Detection of an earlier tubulopathy in diabetic nephropathy among children with normoalbuminuria. Iran J Kidney Dis. 2015 Mar;9(2):126-31.

40 Fu WJ, Xiong SL, Fang YG, Wen S, Chen ML, Deng RT, et al. Urinary tubular biomarkers in short-term type 2 diabetes mellitus patients: a cross-sectional study. Endocrine. 2012 Feb;41(1):82-8.

41 Vijay S, Hamide A, Senthilkumar GP, Mehalingam V. Utility of urinary biomarkers as a diagnostic tool for early diabetic nephropathy in patients with type 2 diabetes mellitus. Diabetes Metab Syndr. 2018 Sep;12(5):64952 . 
42 Lacquaniti A, Donato V, Pintaudi B, Di Vieste G, Chirico V, Buemi A, et al. "Normoalbuminuric" diabetic nephropathy: tubular damage and NGAL. Acta Diabetol. 2013 Dec;50(6):935-42.

43 Satirapoj B. Tubulointerstitial Biomarkers for Diabetic Nephropathy. J Diabetes Res. 2018 Feb;2018:2852398.

44 Titan SM, Vieira JM Jr, Dominguez WV, Moreira SR, Pereira AB, Barros RT, et al. Urinary MCP-1 and RBP: independent predictors of renal outcome in macroalbuminuric diabetic nephropathy. J Diabetes Complications. 2012 Nov-Dec;26(6):546-53.

45 Zhang J, Liu Z, Zhang X, Zhang L, Jin X. Association between urine retinol-binding protein levels and nonalcoholic fatty liver disease: A cross-sectional study in Chinese population. J Clin Lab Anal. 2018 May;32(4):e22359.

46 Fischer JE, Bachmann LM, Jaeschke R. A readers' guide to the interpretation of diagnostic test properties: clinical example of sepsis. Intensive Care Med. 2003 Jul;29(7):1043-51. 